\title{
Brachial plexus tuberculosis: A unique neurological variant of a common clinical disease
}

\author{
Sanjeev Ariyandath Sreenivasan ${ }^{1}$, Sandeep Vaishya ${ }^{2}$, Rana Patir $^{3}$ \\ From ${ }^{1}$ Associate Consultant, ${ }^{2}$ Professor, ${ }^{3}$ Professor and Head, Department of Neurosurgery, Fortis memorial Research Institute, Gurgaon, \\ Haryana, India
}

\begin{abstract}
Isolated primary tuberculous involvement of the brachial plexus has not been reported in the past. Here, we report the case of a 29-year-old male who developed neck pain and radiculopathy for 3 months. Weakness in the left shoulder-elbow ( $2 / 5$ MRC grade) and wrist (4/5 MRC grade) was present with numbness to pain and temperature along the $\mathrm{C} 4 \mathrm{C} 5$ dermatome. Magnetic resonance imaging (MRI) revealed a T2 hyperintense heterogeneously contrast-enhancing lesion involving the upper trunk of the brachial plexus. Under suspicion of malignancy, surgical exploration was undertaken. Intraoperatively, pus was noticed with unhealthy granulation tissue extending along the left-sided C4-5 lamina and transverse process. The frozen section suggested granulomatous infection and histopathology confirmed tuberculosis (TB). After 18 months of antituberculous treatment, the patient gradually recovered complete strength in the left upper limb. Follow-up brachial plexus MRI showed near-complete resolution of the lesion. We describe a unique case of a young patient clinically suggestive of the rapid progressive lesion (mimicking malignancy) affecting brachial plexus, turning out as TB on histopathology.
\end{abstract}

Key words: Brachial plexus, Neuritis, Tuberculosis

$\mathrm{T}$ Tuberculosis (TB) is a common condition affecting a variety of neural structures. TB proved to be the second most common infectious cause of death after coronavirus disease (COVID-19) in the year 2020. According to the Global TB report 2021, $43 \%$ of TB cases were reported from the Southeast Asia region and $26 \%$ were from India. Men represent $56 \%$ of the affected population, while women and children represent 33\% and $11 \%$, respectively. The burden of multidrug-resistant $\mathrm{TB}$ and rifampicin-resistant TB has remained stable at 3-4\% (from 2015 to 2020) [1]. Developing countries have high endemicity for cranial and spinal tuberculous patients. The central nervous system is involved in approximately $10 \%$ of all TB cases [2]. Despite this, isolated involvement of the brachial plexus has not been described in the literature. Non-lesional lumbosacral plexopathy in a patient with pulmonary TB has been reported in the past [3].

\section{CASE REPORT}

We describe a patient with this chronic granulomatous infection of the upper trunk of the brachial plexus, which we believe is

\section{Access this article online}

Received - 14 October 2021

Initial Review - 01 November 2021

Accepted - 29 November 2021

DOI: $10.32677 /$ ijcr.v7i12.3157 the first case of isolated brachial plexus TB to be reported in the literature to date.

A 29-year-old male patient presented with a complaint of neck pain radiating to the left upper limb for 3 months. The pain was localized to the posterior aspect of the left side of the neck, moderate in intensity, pricking type in nature, continuous throughout the day, not aggravated by any particular neck movements, not relieved by any specific neck posture/medications, and gradually began radiating to the left upper limb after 1 week of onset. Two weeks after the onset of pain, he developed rapidly progressive weakness involving the left upper limb. He noticed difficulty in lifting his hand above the shoulder while combing hair, wearing a sweater, lifting heavy objects above head level, and later difficulty in lifting a bucket of water and grasping objects with the left hand. There was no history of similar events in the past.

General examination revealed a conscious, alert, and oriented patient with a pulse rate of $74 / \mathrm{min}$ and blood pressure of $112 / 60 \mathrm{mmHg}$ in the right arm. His body mass index was $20.76 \mathrm{~kg} / \mathrm{m}^{2}$. On clinical examination, bilateral cervical lymph nodes were enlarged, non-tender, and non-matted. The left-sided paravertebral tenderness was noticed adjacent to the $\mathrm{C} 4$ and $\mathrm{C} 5$ levels. No obvious spinal deformity could be identified. The patient had weakness in the left upper limb with a power of $2 / 5$ (MRC grade) at the shoulder and elbow joint and 4/5 at the wrist

Correspondence to: Dr. Sanjeev Sreenivasan, Associate Consultant, Department of Neurosurgery, Fortis Memorial Research Institute, Gurgaon - 122002, Haryana, India. E-mail: sanjeev_ariyan@yahoo.com

(C) 2021 Creative Commons Attribution-NonCommercial 4.0 International License (CC BY-NC-ND 4.0). 
joint. Sensory loss to pain and temperature was 50\% along with C4, C5 dermatomes and biceps, triceps, and supinator reflexes were diminished.

Chest X-ray did not reveal any significant pulmonary findings. Cervical spine X-ray showed a paravertebral opacity near the C4-C5 lateral mass and transverse process region. Magnetic resonance imaging (Figs. 1 and 2) was suggestive of an illdefined heterogeneous contrast-enhancing lesion predominantly involving the upper trunk of the left-sided brachial plexus. A rapidly progressive clinical course was suggestive of malignant etiology and with the intent of obtaining a pathological diagnosis, exploration of the lesion was undertaken.

The left-sided linear paravertebral incision was given at the C4-C5 level. Pus was seen beneath the muscle layer at $\sim 3 \mathrm{~cm}$ depth with unhealthy granulation tissue extending up to the lamina/transverse process of $\mathrm{C} 4-\mathrm{C} 5$ on the left side. Decompression of lesion and evacuation of pus were carried out. Adequate decompression was achieved and no breach of the thecal sac or its contents was noticed. A frozen section of the tissue
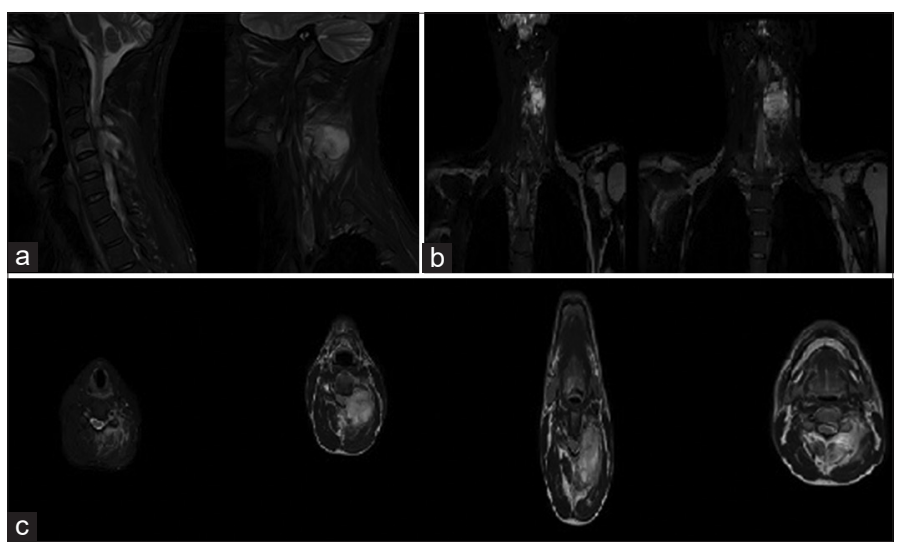

Figure 1: (a) Pre-operative MR images (T2 sagittal) showing lesion localized to the origin of $\mathrm{C} 4-\mathrm{C} 5$ roots and intact anterior spinal column; (b) pre-operative coronal MR images; and (c) pre-operative sequential axial MR images showing location of lesion and relatively well-preserved spinal column
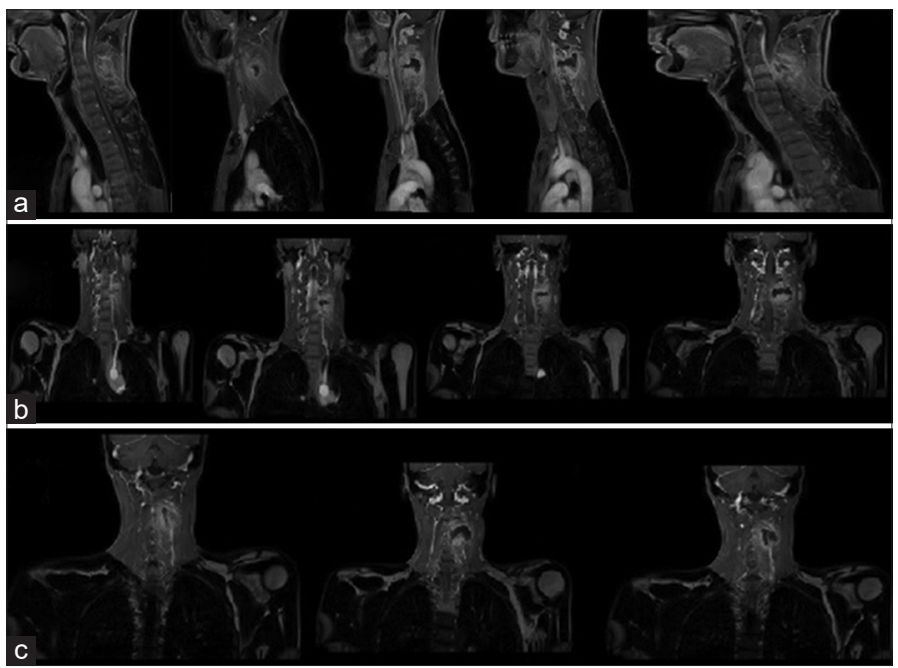

Figure 2: Pre-operative contrast MR images. Row (a) sagittal contrast images showing rim enhancement and row (b and c) coronal contrast images was suggestive of granulomatous infection. GeneXpert from the pus sample was positive. Histopathology revealed necrotizing granulomatous inflammation with a central necrotic core surrounded by epithelioid macrophages, a few multinucleated giant cells, and lymphocytes. Hence, tuberculous etiology was confirmed and malignancy was ruled out.

Based on the frozen section report of granulomatous infection, antituberculous treatment was started with a standard regimen as per the Revised National TB Control Program guidelines [4,5]. Peroral (PO) medications isoniazid ( $5 \mathrm{mg} / \mathrm{kg} /$ day), rifampicin (10 mg/kg/day), pyrazinamide (25 mg/kg/day), ethambutol (15 mg/kg/day), and injectable streptomycin (15 mg/kg/day IM) were given for 2 months. This was followed by a continuation phase of isoniazid (5 $\mathrm{mg} / \mathrm{kg} /$ day $\mathrm{PO}$ ) and rifampicin (10 mg/kg/day PO) for 16 months. The patient showed gradual improvement in motor strength, subsidence of neck swelling, and radiological resolution of spinal compression. Since clinical recovery was maintained at a gradual rate as seen in follow-up outpatient department visits, complete antitubercular treatment (ATT) for 18 months was carried out. Periodic liver function tests were done to rule out the development of ATT-induced hepatitis. Pyridoxine supplementation (25 mg/day PO) was given during the entire treatment duration to prevent ATT-induced peripheral neuropathy. He completed 18 months course of ATT. MR brachial plexus at 4-year follow-up showed good resolution of neural compression and significant subsidence of the lesion (Fig. 3).

\section{DISCUSSION}

There are multiple mimickers of this clinicopathological entity which we considered in our differential diagnosis. There was a clinical possibility of a nerve sheath tumor (schwannoma); however, imaging characteristics suggested otherwise. Cervicothoracic junction TB, acute brachial plexus neuritis, Parsonage-Turner syndrome (PTS), Refsum disease, and hypertrophic polyneuritis of Dejerine are some of the likely differential diagnoses. Cervicothoracic junction TB may present with radiculopathy of the upper limbs [6]. The radiculopathy, lower motor neuron weakness in the distal upper limb muscles, and resultant deformity respond well to debridement and appropriate medical management. Myelopathy is an uncommon feature, in spite of extensive bony destruction. Adult and pediatric-onset cervical TB is differentiated by a frequent occurrence of paraplegia and larger abscesses in the former. Needle electromyography and nerve conduction studies usually show evidence of distal axonal degeneration. In the absence of radiological indicators, various differentials need to be considered.

Acute brachial plexus neuritis causes painful restriction of the upper limb movements. Involvement of the brachial plexus in various infective diseases has been described [7]. Seo et al. demonstrated a patient with Streptococcus agalactiae infection causing pyogenic shoulder joint arthritis and subsequent brachial plexus neuritis. They postulated an immune reaction to the streptococcus antigen as the probable etiological mechanism. 


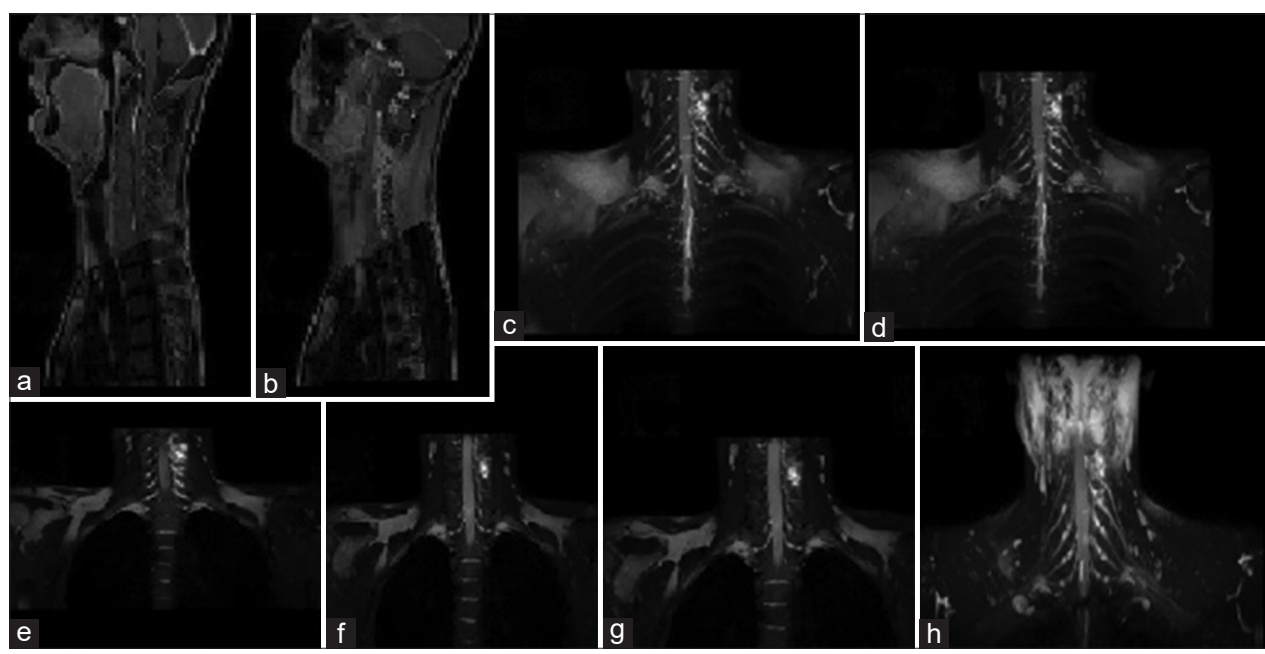

Figure 3: Post-operative MR brachial plexus. (a and b) Sagittal contrast images showing no residual lesion/compression of nerve roots and (c-h) coronal contrast images showing resolved compression with scar tissue

PTS is a similar condition with sudden onset pain and restriction of shoulder movements followed by atrophy of the upper limb muscles. Possible etiology includes trauma, recent immunization, surgery on the brachial plexus region, preceding viral infection, unusual strenuous physical activity, and the predisposing connective tissue or autoimmune disorders. Physiological recovery is seen in $70-90 \%$ of patients, while $10-20 \%$ have impaired exercise tolerance and persistent pain. The use of intravenous immunoglobulins as a therapeutic tool for PTS is under investigational stages.

Refsum disease and interstitial hypertrophic polyneuritis of Dejerine present a similar clinical picture. Joffroy in 1879 first elucidated neuralgia amyotrophy of the brachial plexus in a patient with sudden onset restriction of shoulder movement [8]. Mononeuritis multiplex with brachial plexus predilection is a condition characterized by sharp stabbing pain, weakness, and atrophy involving the shoulder. Taylor described this entity with a heredofamilial background [9]. The prognosis of neurological recovery in patients with brachial plexus neuritis is good with $75-80 \%$ recovery in 2 years and almost complete recovery in 3 years.

In our patient, GeneXpert of the pus sample was positive. It has been studied that GeneXpert can be $100 \%$ specific and 90-95\% sensitive for diagnosing TB [10]. Histopathology is considered at least $85 \%$ specific and $93-94 \%$ sensitive as compared to TB-polymerase chain reaction [11]. TB can predispose to neuropathy through various mechanisms vis-à-vis spinal tuberculomas, meningeal TB, peripheral neuropathy due to immune-related mechanisms, and secondary to antitubercular therapy.

Pyridoxine deficiency is common in patients using combinations of pyrazinamide and isoniazid. This usually manifests will loss of proprioception and vibration sense. Hence, we supplement ATT with oral pyridoxine till the entire course is complete. Multidrug antitubercular chemotherapy is the mainstay of treatment. Debridement and surgical stabilization are required only in certain situations such as progressive neurological deficit and worsening mechanical construct stability. The standard ATT regime we follow comprises 2 months of isoniazid $(\mathrm{H})$, rifampicin $(\mathrm{R})$, pyrazinamide $(\mathrm{Z})$, ethambutol (E), and streptomycin (S) followed by HR for 16 months. A Medical Research Council reported similar functional outcomes in patients with spinal TB undergoing chemotherapy, debridement, and/or fusion [12-14]. In our patient, debridement and evacuation of pus enabled early decompression of neural elements.

\section{CONCLUSION}

TB is a chronic granulomatous disease that can involve any system or component of the human body. Isolated involvement of the brachial plexus has not been reported in the medical literature. We describe this unique presentation of weakness in the upper limb secondary to tubercular involvement of the upper trunk of the brachial plexus. Debridement and evacuation of pus for relieving symptoms of compression of neurovascular structures must be timely done. A complete course of antitubercular chemotherapy is vital for a good neurological outcome.

\section{REFERENCES}

1. Global Tuberculosis Report 2021; 2021. Available from: https://www.who. int/teams/global-tuberculosis-programme/tb-reports/global-tuberculosisreport-2021 [Last accessed on 2021 Nov 01].

2. Muralidharan V, Nair BR, Rajshekhar V. Changing trends of presentation of central nervous system tuberculosis: Relative prevalence of cranial and spinal tuberculosis and drug resistance patterns. Neurol India 2019;67:792-6.

3. Stoeckli TC, Mackin GA, de Groote MA. Lumbosacral plexopathy in a patient with pulmonary tuberculosis. Clin Infect Dis 2000;30:226-7.

4. Khadilkar SV, Kadam ND, Kulkarni RV, Meshram CM, Meshram AR, Patel BA, et al. Guidelines versus ground lines: Tuberculosis of the central nervous system. Neurol India 2019;67:787-91.

5. Guidelines: Central TB Division; 2021. Available from: https://tbcindia.gov. in/index 1 .php?lang=1\&level=1\&sublinkid=4571\&lid=3176 [Last accessed on 2021 Oct 26].

6. Gopalakrishnan D, Krishna KN. Cervicothoracic spinal tuberculosis presenting as radiculopathy. Neurol India 2002;50:93-4.

7. Seo JY, Lee JY, Hong BY. Brachial plexus neuritis associated with Streptococcus agalactiae: A case report. Ann Rehabil Med 2014;38:563-7.

8. Verma R, Sharma P, Khurana N. Neuralgia amyotrophy associated with 
dengue fever: Case series of three patients. J Postgrad Med 2011;57:329-31.

9. Taylor AR. Heredofamilial mononeuritis multiplex with brachial predilection. Brain 1960;83:113-37.

10. Solanki AM, Basu S, Biswas A, Roy S, Banta A. Sensitivity and specificity of gene Xpert in the diagnosis of spinal tuberculosis: A prospective controlled clinical study. Global Spine J 2020;10:553-8.

11. Courcoul A, Moyen JL, Brugère L, Faye S, Hénault S, Gares H, et al. Estimation of sensitivity and specificity of bacteriology, histopathology and PCR for the confirmatory diagnosis of bovine tuberculosis using latent class analysis. PLoS One 2014;9:e90334.

12. A controlled trial of six-month and nine-month regimens of chemotherapy in patients undergoing radical surgery for tuberculosis of the spine in Hong Kong. Tenth report of the medical research council working party on tuberculosis of the spine. Tubercle 1986;67:243-59.

13. CDC. Controlling Tuberculosis in the United States: Recommendations from the American Thoracic Society, CDC, and the Infectious Diseases
Society of America, MMWR No. RR-12. Vol. 54. Atlanta, Georgia, United States: CDC; 2005. Available from: https://www.cdc.gov/mmwr/preview/ mmwrhtml/rr5412a1.htm. [Last accessed on 2021 Sep 28].

14. CDC. Treatment of Tuberculosis, MMWR No. RR-11. Vol. 52. Atlanta, Georgia, United States: American Thoracic Society, CDC, and Infectious Diseases Society of America; 2003. Available from: https://www.cdc.gov/ $\mathrm{mmwr} / \mathrm{preview} / \mathrm{mmwrhtml} / \mathrm{rr} 5211 \mathrm{a} 1 . \mathrm{htm}$. [Last accessed on 2021 Oct 17].

Funding: None; Conflicts of Interest: None Stated.

How to cite this article: Sreenivasan SA, Vaishya S, Patir R. Brachial plexus tuberculosis: A unique neurological variant of a common clinical disease. Indian J Case Reports. 2021;7(12):522-525. 